\section{(C) OPEN ACCESS}

\title{
Clinical outcomes in patients switched from adalimumab to baricitinib due to non-response and/ or study design: phase III data in patients with rheumatoid arthritis
}

\author{
Yoshiya Tanaka, ${ }^{1}$ Bruno Fautrel, ${ }^{2}$ Edward C Keystone, ${ }^{3,4}$ Robert A Ortmann, ${ }^{5}$ Li Xie, ${ }^{5}$ \\ Baojin Zhu, ${ }^{5}$ Maher Issa, ${ }^{5}$ Himanshu Patel, ${ }^{5}$ Carol L Gaich, ${ }^{5}$ Stephanie de Bono, \\ Terence P Rooney, ${ }^{5}$ Peter C Taylor ${ }^{\circ}$
}

\begin{abstract}
Handling editor Josef $S$ Smolen

- Additional material is published online only. To view please visit the journal online (http://dx.doi.org/10.1136/ annrheumdis-2018-214529).
\end{abstract}

For numbered affiliations see end of article.

Correspondence to Dr Yoshiya Tanaka, First Department of Internal Medicine, University of Occupational and Environmenta Health, Japan, Kitakyushu 8078555, Japan;

tanaka@med.uoeh-u.ac.jp

Received 4 October 2018 Revised 8 March 2019 Accepted 27 March 2019 Published Online First 30 April 2019

\section{Check for updates}

(c) Author(s) (or their employer(s)) 2019. Re-use permitted under CC BY-NC. No commercial re-use. See rights and permissions. Published by BMJ.

To cite: Tanaka $Y$, Fautrel B, Keystone EC, et al. Ann Rheum Dis 2019:78:890-898.

\section{ABSTRACT}

Objective To evaluate clinical outcomes in patients who changed treatment from adalimumab to baricitinib, an oral Janus kinase (JAK)1/JAK2 inhibitor, during a phase III programme.

Methods In phase III RA-BEAM, patients were randomised 3:3:2 to placebo, baricitinib 4 mg once daily, or adalimumab $40 \mathrm{mg}$ biweekly. At week 16 or subsequent visits, non-responders were rescued to open-label baricitinib $4 \mathrm{mg}$. At week 52, patients could enter a long-term extension (LTE) and continue on baricitinib or switch from adalimumab to baricitinib 4 mg with no adalimumab washout period. Percentage of patients achieving low disease activity and remission were assessed, along with physical function, patient's assessment of pain, and safety.

Results Thirty-five (7\%) baricitinib-treated and 40 $(12 \%)$ adalimumab-treated patients were rescued to baricitinib in RA-BEAM; 78\% (381/487) of baricitinibtreated and $72 \%(238 / 330)$ of adalimumab-treated patients who were not rescued in RA-BEAM, entered the LTE and continued/were switched to baricitinib. In both baricitinib-rescued and adalimumab-rescued patients, there were significant improvements in all measures up to 12 weeks after rescue compared with the time of rescue. Patients who switched from adalimumab to baricitinib showed improvements in disease control through 12 weeks in the LTE. Exposure-adjusted incidence rates for treatment-emergent adverse events (TEAEs) and infections, including serious events, were similar for patients who switched from adalimumab to baricitinib and those who continued on baricitinib.

Conclusions Switching from adalimumab to baricitinib (without adalimumab washout) was associated with improvements in disease control, physical function and pain during the initial 12 weeks postswitch, without an increase in TEAEs, serious adverse events or infections.

\section{Trial registration numbers NCT01710358,}

NCT01885078.

\section{INTRODUCTION}

The combined use of conventional synthetic disease-modifying antirheumatic drugs, targeted synthetic disease-modifying antirheumatic drugs (tsDMARDs) or biological disease-modifying antirheumatic drugs (bDMARDs), as well as the

\section{Key messages}

What is already known about this subject?

- In patients who do not respond adequately to first-line therapies or who discontinue initial treatment due to intolerance, alternative treatment strategies are used, including adding or switching to other disease-modifying antirheumatic drugs.

- Because switching therapies is common in clinical practice, the safety and efficacy of new therapies should be assessed when used as replacement treatment following initial regimens.

\section{What does this study add?}

- In the phase III RA-BEAM study, patients taking adalimumab or baricitinib could be rescued to baricitinib at week 16 or later due to inadequate response; at the end of RA-BEAM, and on entry to a long-term extension (LTE) study, patients were switched/continued to baricitinib with no adalimumab washout period.

- In both baricitinib-rescued and adalimumabrescued patients, there were significant improvements in all efficacy and patientreported outcome measures up to 12 weeks after rescue compared with the time of rescue. Patients who switched from adalimumab to baricitinib showed improvements in disease control through 12 weeks in the LTE.

- Incidence rates for adverse events and infections, including serious events, were similar for patients who switched from adalimumab to baricitinib and for those who continued baricitinib. 
Key messages

How might this impact on clinical practice or future developments?

- Transition from adalimumab to baricitinib does not require lengthy washout from the prior treatment in patients with rheumatoid arthritis and is associated with improvements in clinical disease control, with acceptable safety.

antirheumatic drugs (DMARDs). Because switching therapies is common in clinical practice, the safety and efficacy of new therapies should be assessed when used as replacement treatment following initial regimens.

Baricitinib is a recently developed tsDMARD and an oral selective inhibitor of Janus kinase (JAK) 1 and JAK2, which belong to a family of protein tyrosine kinases that mediate signal transduction for a variety of cytokines involved in inflammatory conditions, including RA. ${ }^{45}$ Baricitinib is approved for the treatment of moderately to severely active RA in adults in over 50 countries, including European countries, the USA and Japan. The 52-week RA-BEAM study (NCT01710358) of methotrexate-inadequate responder (MTX-IR) patients with active RA showed that baricitinib $4 \mathrm{mg}$ demonstrated superior efficacy compared with placebo and adalimumab, a tumour necrosis factor (TNF) inhibitor, based on $20 \%$ response according to the criteria of the American College of Rheumatology at 12 weeks, as well as secondary measures, including mean change in disease activity score for 28 joints with the use of high-sensitivity $\mathrm{C}$ reactive protein and percent of patients achieving LDA based on Simplified Disease Activity Score (SDAI) and Clinical Disease Activity Score (CDAI). ${ }^{6}$

During RA-BEAM, non-responders were rescued to baricitinib, and at the completion of RA-BEAM, patients had the option to enrol in a long-term extension (LTE) study, RA-BEYOND (NCT01885078), in which all patients were switched to baricitinib. The objectives of this analysis were to evaluate the efficacy, physical function, pain and safety in two groups of patients from RA-BEAM: patients taking adalimumab or baricitinib who were rescued to baricitinib during RA-BEAM and those who were switched to baricitinib on entering the LTE study RA-BEYOND.

\section{METHODS}

The eligibility criteria for the originating study, RA-BEAM, have been previously published. ${ }^{6}$ In brief, RA-BEAM included patients aged $\geq 18$ years with a confirmed diagnosis of active RA, defined as $\geq 6 / 68$ tender and $\geq 6 / 66$ swollen joints, serum high-sensitivity $\mathrm{C}$ reactive protein (hsCRP) $\geq 6 \mathrm{mg} / \mathrm{L}$ and $\geq 3$ joint erosions (patients with one to two erosions could enrol if they were rheumatoid factor or anti-citrullinated protein antibodies positive). Patients with prior bDMARD therapy were excluded from RA-BEAM. All patients completing the 52 weeks of RA-BEAM, regardless of rescue status, were eligible to enrol in RA-BEYOND. Safety-related exclusion criteria for RA-BEYOND included having significant medical issues that developed during RA-BEAM that, in the opinion of the investigator, posed an unacceptable risk to the patient if baricitinib/study drug continued to be administered.

\section{Study design and treatments}

RA-BEAM was a 52 week, double-blind, placebo-controlled and active-controlled phase III study in adult MTX-IR patients with active RA (online supplementary figure S1). Patients were randomised 3:3:2 to receive oral placebo once daily, oral baricitinib $4 \mathrm{mg}$ once daily or subcutaneous adalimumab $40 \mathrm{mg}$ biweekly. Patients continued receiving background MTX (stable dose of 7.5-25 mg/week). At week 16 in RA-BEAM, non-responders (lack of improvement of $\geq 20 \%$ in tender joint and swollen joint count at both weeks 14 and 16 compared with baseline) received rescue treatment (baricitinib $4 \mathrm{mg}$ ). After week 16, patients received rescue treatment at the investigator's discretion on the basis of tender and swollen joint counts. Rescued patients received open-label baricitinib once daily, but no biweekly subcutaneous injection, for the remainder of the study. From the time of rescue (but not before), background corticosteroids (maximum dose of prednisone $10 \mathrm{mg}$ daily or equivalent), non-steroidal anti-inflammatories and analgesics could be added or increased in dose. Rescuing all groups to baricitinib (including those already receiving baricitinib with inadequate response) therefore increased baricitinib exposures for safety evaluation by regulators, while still offering patients some escalation of (background) treatment. At week 24, patients receiving placebo were switched to baricitinib, unaware of the change in treatment. Rescued/switched patients and their investigators remained blinded to original treatment assignment.

RA-BEYOND is a phase III LTE study of the efficacy and safety of baricitinib in patients with RA (study duration up to 7 years). On entering RA-BEYOND, all patients from RA-BEAM continued on baricitinib $4 \mathrm{mg}$ once daily or switched from adalimumab therapy to baricitinib $4 \mathrm{mg}$ QD. All patients entering RA-BEYOND remained blinded to original treatment. No washout period of adalimumab occurred in patients who were rescued during RA-BEAM or switched at the entry to RA-BEYOND.

The current analysis includes two separate patient populations: (1) rescued patients in RA-BEAM: patients who were rescued from blinded baricitinib or adalimumab to open-label baricitinib between weeks 16 and 24 due to non-response (data after rescue from placebo are presented in the online supplementary material); and (2) switched patients in RA-BEYOND: patients who were not rescued in RA-BEAM, who were originally randomised to baricitinib and continued baricitinib or who switched from adalimumab to baricitinib at the entry of RA-BEYOND, and entered RA-BEYOND at least 24 weeks before the data cut-off date (1 September 2016). In RA-BEAM, efficacy data collection (visits) for rescued patients occurred at weeks 16 (first rescue opportunity), 20, 24, 28, 32, 40 and 52. Efficacy data are not presented in patients who were rescued after week 24 because data acquisition (visits) occurred less frequently than in patients rescued earlier.

RA-BEAM and RA-BEYOND were conducted in accordance with the principles of the Declaration of Helsinki and Good Clinical Practice Guidelines and approved by each centre's institutional review board or ethics committee. All patients provided written informed consent. The studies were designed by the sponsors, Eli Lilly and Company and Incyte Corporation, with input from an academic advisory board in which non-Lilly authors of this article participated. All authors participated in the preparation and review of this manuscript and approved the final version.

\section{Patient involvement}

This research was done without patient involvement. Patients were not invited to comment on the study design and were not consulted to develop patient relevant outcomes or interpret the 
results. Patients were not invited to contribute to the writing or editing of this document for readability or accuracy.

\section{Efficacy and patient-reported outcomes}

Efficacy assessments included the proportion of patients achieving LDA and remission based on CDAI (LDA $\leq 10$, remission $\leq 2.8$ ), SDAI (LDA $\leq 11$, remission $\leq 3.3$ ) and disease activity score using 28 -joint counts with erythrocyte sedimentation rate (DAS28-ESR; LDA $\leq 3.2$, remission $<2.6$ ), as well as change from baseline of these measures. Patient-reported outcomes (PROs) included assessment of physical function using the Health Assessment Questionnaire Disability Index (HAQDI) and patient's assessment of pain $(0-100 \mathrm{~mm}$, visual analogue scale). Continuous data for the individual acute phase markers (erythrocyte sedimentation rate and hsCRP) are also presented. In patients who were rescued during RA-BEAM, efficacy and PROs are reported up to 12 weeks after rescue. In patients who switched to baricitinib on entry to RA-BEYOND, outcomes were reported at the baseline of the LTE and up to 24 weeks after entry into the LTE.

\section{Safety}

Treatment-emergent adverse events (TEAEs), including infections, specifically herpes zoster and gastrointestinal disorders; discontinuations; and serious adverse events (SAEs), including serious infections, were assessed. Safety was assessed through 24 weeks after the transition to baricitinib $4 \mathrm{mg}$.

\section{Statistical analyses}

Statistical analyses were conducted for patients' clinical outcomes and PROs before and after treatment change due to rescue or switch. In rescued patients, comparisons were between the time of rescue and 4, 8 and 12 weeks after rescue. In patients who switched treatment on entry to the LTE, comparisons were between the time of switch and 4, 12 and 24 weeks after switch. For continuous measures, a mixed model for repeated measures approach was used with treatment, visit and treatment-by-visit interaction included. For categorical measures, the proportion of patients who achieved LDA and remission was summarised; non-responder imputation was applied for missing data and after permanent discontinuation of the study drug. Safety data are expressed as percentages and exposure-adjusted incidence rates (EAIRs) up to 24 weeks for the rescued and switched patients separately. Due to the non-randomised nature of this analysis, no between-group statistical comparisons were made.

\section{RESULTS}

\section{Patients}

A total of 1307 patients were randomised and 1305 (488 placebo, 487 baricitinib $4 \mathrm{mg}$ and 330 adalimumab) patients received treatment. Between weeks 16 and 24, 35 (7\%) patients treated with baricitinib and $40(12 \%)$ treated with adalimumab were rescued to open-label baricitinib in RA-BEAM. The majority of rescued patients completed RA-BEAM through week 52: 25 patients (74\%) treated with baricitinib-to-baricitinib and 35 patients (90\%) treated with adalimumab-to-baricitinib. A total of $78 \%(381 / 487)$ of baricitinib-treated patients and $72 \%(238 / 330)$ of adalimumab-treated patients were not rescued in RA-BEAM and entered the LTE $\geq 24$ weeks before the data cut-off date. The disposition of patients is shown in online supplementary figure S2. Baseline demographics and clinical characteristics of all patients and patients rescued during RA-BEAM are listed in table 1. Overall, patients had similar demographics across the treatment groups; $80 \%$ of rescued

Table 1 Characteristics and disease activity at baseline of RA-BEAM for the overall baricitinib-treated and adalimumab-treated patients and for patients rescued to baricitinib between weeks 16 and 24

\begin{tabular}{|c|c|c|c|c|}
\hline & \multicolumn{2}{|c|}{ Overall patients in RA-BEAM } & \multicolumn{2}{|c|}{ Patients rescued to baricitinib } \\
\hline & $\begin{array}{l}\text { Baricitinib } \\
4 \mathrm{mg}(\mathrm{n}=487)\end{array}$ & $\begin{array}{l}\text { Adalimumab } \\
(n=330)\end{array}$ & $\begin{array}{l}\text { Baricitinib } \\
4 \mathrm{mg}(\mathrm{n}=35)\end{array}$ & $\begin{array}{l}\text { Adalimumab } \\
(\mathrm{n}=40)\end{array}$ \\
\hline Age, years & $53.5(12)$ & $52.9(12)$ & $52.3(12)$ & $51.9(14)$ \\
\hline Female, n (\%) & $375(77)$ & $251(76)$ & $30(86)$ & $31(78)$ \\
\hline Duration of RA, ${ }^{*}$ years & $8.7(8.6)$ & $8.3(7.9)$ & $8.0(7.8)$ & $7.0(6.2)$ \\
\hline ACPA positive, $\mathrm{n}(\%)$ & $427(88)$ & $295(89)$ & $28(80)$ & $37(93)$ \\
\hline RF positive, $n(\%)$ & $439(90)$ & $301(91)$ & $30(86)$ & $38(95)$ \\
\hline Swollen joint count, of 66 & $15(8)$ & $15(9)$ & $19(10)$ & $19(12)$ \\
\hline Tender joint count, of 68 & $23(13)$ & $23(14)$ & $25(13)$ & $23(11)$ \\
\hline hsCRP, mg/L & $22(23)$ & $22(21)$ & $20(20)$ & $31(29)$ \\
\hline ESR, mm/hour & $49(26)$ & $48(26)$ & $46(25)$ & $56(30)$ \\
\hline DAS28-hsCRP & $5.8(0.9)$ & $5.8(0.9)$ & $6.0(0.9)$ & $6.1(0.9)$ \\
\hline DAS28-ESR & $6.5(0.9)$ & $6.4(1.0)$ & $6.7(1.0)$ & $6.8(1.0)$ \\
\hline CDAl & $38(12.0)$ & $38(13)$ & $43(13.6)$ & $42(13.0)$ \\
\hline SDAI & $40(12.7)$ & $40(13.4)$ & $45(14.2)$ & $45(14.0)$ \\
\hline MTX average weekly dose, mg & $14.9(4.6)$ & $14.6(4.4)$ & $15.5(5.3)$ & $14.4(4.2)$ \\
\hline HAQ-DI & $1.57(0.68)$ & $1.59(0.70)$ & $1.69(0.64)$ & $1.75(0.64)$ \\
\hline Patient's assessment of pain & $61.8(21.8)$ & $61.0(22.7)$ & $67.6(23.2)$ & $67.1(20.9)$ \\
\hline
\end{tabular}

Data are mean (SD) unless otherwise stated.

*Time from RA diagnosis.

ACPA, anti-citrullinated protein antibodies; CDAl, Clinical Disease Activity Index; DAS28-ESR, disease activity score using 28-joint count with erythrocyte sedimentation rate; DAS28-hsCRP, disease activity score for 28 joints with the use of high-sensitivity C reactive protein; ESR, erythrocyte sedimentation rate; HAQ-DI, Health Assessment Questionnaire Disability Index; MTX, methotrexate-inadequate responder; RA, rheumatoid arthritis; RF, rheumatoid factor; SDAl, Simplified Disease Activity Index; hsCRP, highsensitivity $C$ reactive protein. 
Table 2 Change in efficacy and patient-reported outcomes 4, 8 and 12 weeks after rescue in rescued patients from RA-BEAM

\begin{tabular}{|c|c|c|c|c|c|c|c|c|}
\hline & \multicolumn{4}{|c|}{ Baricitinib to baricitinib $(n=35)$} & \multicolumn{4}{|c|}{ Adalimumab to baricitinib $(n=40)$} \\
\hline & \multirow{2}{*}{$\begin{array}{l}\text { Score at time } \\
\text { of rescue }\end{array}$} & \multicolumn{3}{|c|}{ Change from rescue visit } & \multirow{2}{*}{$\begin{array}{l}\text { Score at time } \\
\text { of rescue }\end{array}$} & \multicolumn{3}{|c|}{ Change from rescue Visit } \\
\hline & & Week $4(n=34)$ & Week $8(n=33)$ & Week $12(n=30)$ & & Week $4(n=40)$ & Week 8 ( $n=39)$ & Week $12(n=33)$ \\
\hline DAS28-ESR & $6.1(1.3)$ & $-0.93(0.18)^{* * *}$ & $-1.51(0.20)^{* * *}$ & $-1.73(0.22)^{* * *}$ & $6.2(1.3)$ & $-1.38(0.17)^{* * *}$ & $-1.69(0.19)^{* * *}$ & $-1.92(0.21)^{* * *}$ \\
\hline CDAI & $39.3(15.2)$ & $-14.1(2.1)^{* * *}$ & $-20.5(2.2)^{* * *}$ & $-22.3(2.3)^{* * *}$ & $36.4(14.5)$ & $-14.8(2.0)^{* * *}$ & $-17.8(2.0)^{* * *}$ & $-20.2(2.2)^{* * *}$ \\
\hline SDAI & $40.8(16.0)$ & $-14.1(2.3)^{* * *}$ & $-21.0(2.3)^{\star * *}$ & $-23.1(2.5)^{* * *}$ & $38.9(14.5)$ & $-16.2(2.1)^{* * *}$ & $-19.2(2.2)^{* * *}$ & $-21.8(2.3)^{* * *}$ \\
\hline $\begin{array}{l}\text { Patient's assessment } \\
\text { of pain }\end{array}$ & $53.8(21.0)$ & $-13.1(3.8)^{* * *}$ & $-13.8(3.9)^{* * *}$ & $-18.3(4.0)^{* * *}$ & $56.4(25.4)$ & $-14.5(3.5) * * *$ & $-21.5(3.6)^{* * *}$ & $-24.1(3.7)^{* * *}$ \\
\hline HAQ-DI & $1.42(0.72)$ & $-0.19(0.08)^{*}$ & $-0.28(0.08)^{* * *}$ & $-0.34(0.09)^{* * *}$ & $1.46(0.66)$ & $-0.37(0.07) * * *$ & $-0.47(0.08)^{* * *}$ & $-0.49(0.09)^{* * *}$ \\
\hline CRP, mg/L & $14.9(23.5)$ & $-0.45(4.35)$ & $-4.79(4.49)$ & $-8.50(5.04)$ & $25.3(29.8)$ & $-13.50(4.00)^{* * *}$ & $-14.10(4.13)^{* * *}$ & $-16.00(4.69)^{* * *}$ \\
\hline $\mathrm{ESR}, \mathrm{mm} / \mathrm{h}$ & $36.5(27.7)$ & $0.71(3.40)$ & $-4.49(4.16)$ & $-5.05(4.76)$ & $51.9(35.4)$ & $-16.60(3.16)^{* * *}$ & $-21.80(3.85)^{* * *}$ & $-19.06(4.48)^{* * *}$ \\
\hline
\end{tabular}

Score at the time of rescue data are mean (SD); change from baseline data is LSM (SE).

${ }^{*} p \leq 0.05 ;{ }^{* *} p \leq 0.01 ;{ }^{* * *} p \leq 0.001$ from within-group mean change from the last visit prior to rescue using MMRM.

CDAl, Clinical Disease Activity Index;CRP, C reactive protein; DAS28-ESR, disease activity score using 28-joint count with erythrocyte sedimentation rate;ESR, erythrocyte sedimentation rate; HAQ-

DI, Health Assessment Questionnaire Disability Index; LSM, least squares mean; MMRM, mixed-effects model repeated measures; SDAl, Simplified Disease Activity Index.

patients were female, the mean age was 51.8 years, and the mean RA duration was 7.9 years (table 1 ).

\section{Efficacy and PROs of rescued patients}

In patients rescued in RA-BEAM, both patients treated with baricitinib and patients treated with adalimumab showed significant improvements in all measures at weeks 4, 8 and 12 after rescue compared with values at the time of rescue (table 2). Online supplementary table 1 shows results of placebo patients who were rescued. At the time of rescue, no patients were in LDA or remission based on CDAI or SDAI; 1 (3\%) baricitinib patient was in LDA and $1(3 \%)$ adalimumab patient was in LDA and remission based on DAS28-ESR (figure 1). By week 12 after rescue, $21 \%, 33 \%$ and $33 \%$ of baricitinib and $17 \%$, $37 \%$ and $43 \%$ of adalimumab patients had reached LDA based on DAS28-ESR, CDAI, and SDAI, respectively (figure 1). Acute phase markers decreased significantly in patients rescued from adalimumab to baricitinib, but not in those remaining on baricitinib (table 2). Rescued patients showed significant improvements in pain and physical function 4 weeks after rescue, which were sustained through 12 weeks (table 2). After week 24, an additional eight baricitinib patients and 11 adalimumab patients were rescued. These patients were not included in this study, but a sensitivity analysis using all patients rescued between weeks 16 and 52 showed consistent findings to the patients rescued from weeks 16 to 24 (data not shown).

\section{Efficacy and PROs of switched patients in the LTE}

Patients who switched from adalimumab to baricitinib maintained disease response in the LTE, with improvement in the first 12 weeks post-switch. Patients who continued treatment with baricitinib maintained disease response (figure 2). In patients initially treated with adalimumab in RA-BEAM, mean CDAI, SDAI and DAS28-ESR showed statistically significant improvement through 24 weeks of the LTE after switching to baricitinib $4 \mathrm{mg}$ (figure 2). Overall, the percentage of switched patients who achieved LDA based on CDAI and SDAI increased from the time of switch (entry to RA-BEYOND) to week 24 for both patients treated with baricitinib-to-baricitinib and patients treated with adalimumab-to-baricitinib (figure 3 ).

During the LTE improvements in physical function and pain were maintained in patients continuing baricitinib and patients who switched from adalimumab to baricitinib; by week 24 of the LTE, both groups had similar outcomes related to pain and physical function (figure 2).

\section{Safety}

In rescued patients, EAIRs for infections, SAEs and AEs leading to discontinuation were numerically higher in patients who continued baricitinib. The most common TEAEs were infections and gastrointestinal disorders (table 3). The most common infections were nasopharyngitis $(n=2$ for baricitinib-to-baricitinib and $n=2$ for adalimumab-to-baricitinib) and urinary tract infection ( $\mathrm{n}=2$ for baricitinib-to-baricitinib). In switched patients in the LTE, EAIRs for TEAEs, infections and serious infections were similar in patients who switched from adalimumab to baricitinib and patients who continued baricitinib (table 3 ).

\section{DISCUSSION}

On treatment failure with adalimumab, patients rescued with baricitinib $4 \mathrm{mg}$ showed sustained and clinically relevant improvements in efficacy and PROs, representing measures that are important to patients. After switching from adalimumab to baricitinib on entering the LTE without adalimumab washout, disease control and maintenance in PROs were sustained through 24 weeks postswitch. There was no increase in overall TEAEs, SAEs or infections after transitioning to baricitinib, when patients could have still been exposed to therapeutic levels of adalimumab during the first 12 weeks after the treatment change. Safety findings were consistent with the known safety profile of baricitinib.

Within the RA patient population, the magnitude of efficacy responses to therapeutic intervention is heterogeneous. The contemporary treatment goal is to achieve LDA or remission. ${ }^{3}$ In the absence of a satisfactory clinical response, in patients initiated on MTX, options exist to titrate the dose upwards or change to parenterally administered drugs. In contrast, for patients on targeted bDMARD and tsDMARD therapies, there are limited options for dose titration; therefore, current practice is most often to switch between different therapies, either within class, in the case of biologic TNF inhibitors, or from one mechanism of action to another. The data emerging from the present analyses are therefore of contemporary relevance with respect to two sets of patients: first, patients requiring rescue to a new drug due to lack of clinical response, and second, patients who 


\section{Baricitinib to Baricitinib}
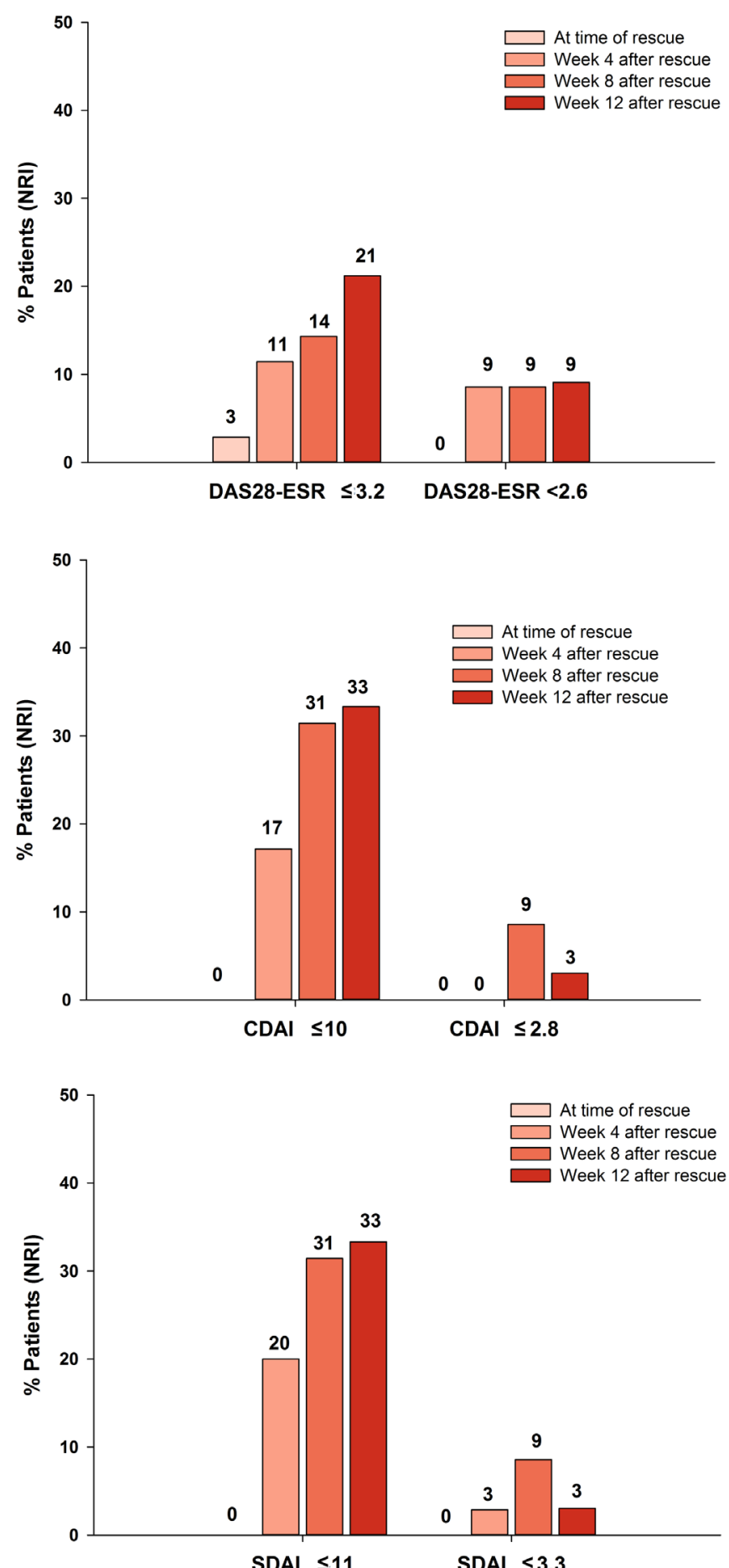

Adalimumab to Baricitinib
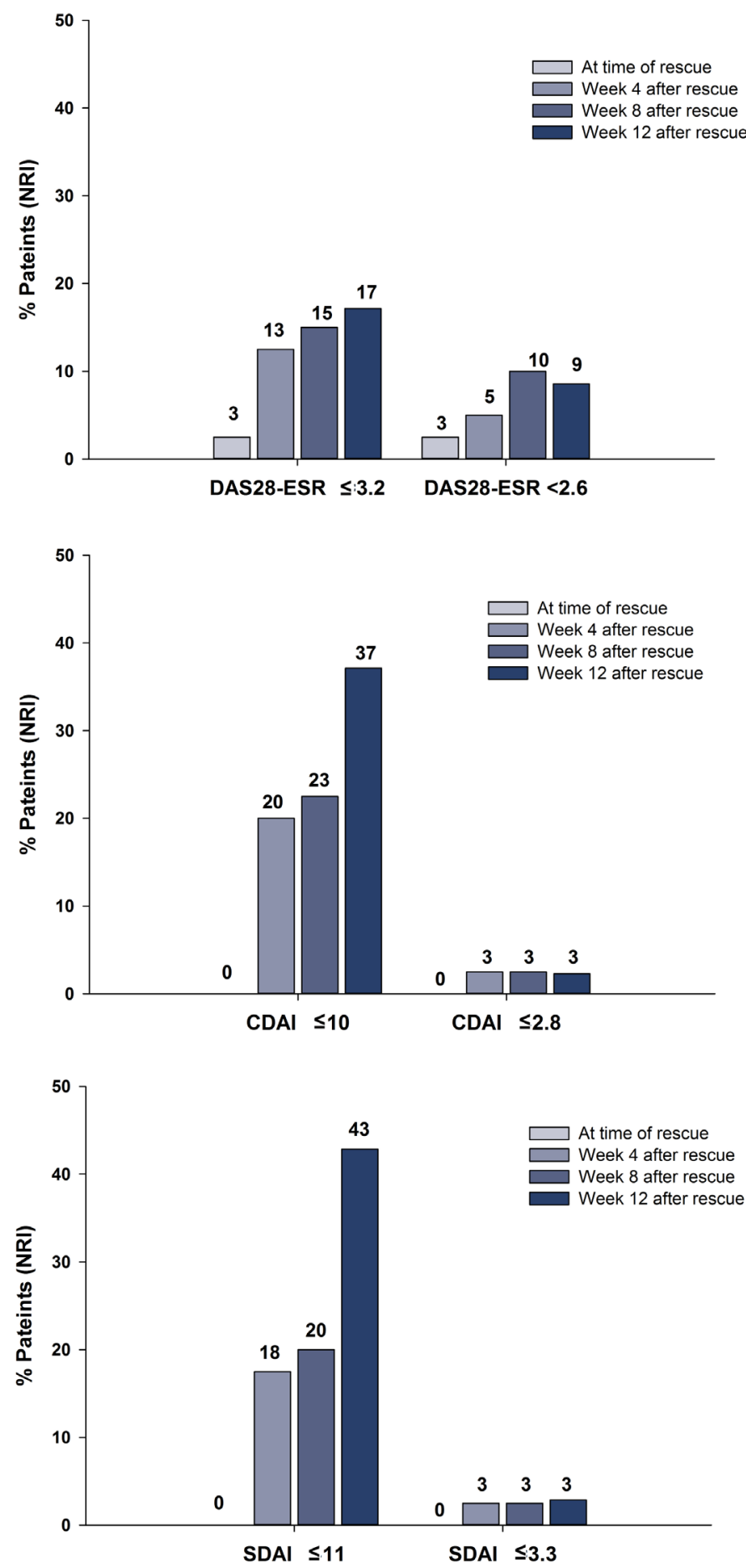

Figure 1 Low disease activity and remission at the time of rescue and 4, 8 and 12 weeks after rescue in rescued patients from RA-BEAM. NRI was used for missing data imputation. CDAI, clinical disease activity index; DAS28-ESR, disease activity score using 28-joint count with erythrocyte sedimentation rate; NRI, non-responder imputation; SDAl, simplified disease activity index.

have a clinical response but choose to switch therapies for other reasons.

The proportion of patients with RA with inadequate response or intolerance to TNF inhibitors is increasing with wider use of these agents. ${ }^{7}$ The phase III RA-BEACON study showed that baricitinib is an effective and well-tolerated once daily oral treatment option for such patients. ${ }^{8}$ Phase III trials of novel targeted DMARDs in TNF-IR RA patients typically require discontinuation of TNF inhibitors prior to enrolment. Commonly, the required period of discontinuation for the previous TNF inhibitor is based on a multiple of half-lives for the specific agent. This results in lengthy washout periods prior to commencing the novel investigational treatment. In an effort to more closely reflect routine clinical practice, RA-BEACON (a phase III randomised controlled trial of baricitinib in patients with inadequate response to TNF inhibitors) adopted a more pragmatic minimum of 4 weeks' discontinuation for prior TNF inhibitors before patients could enter the study screening period. The 

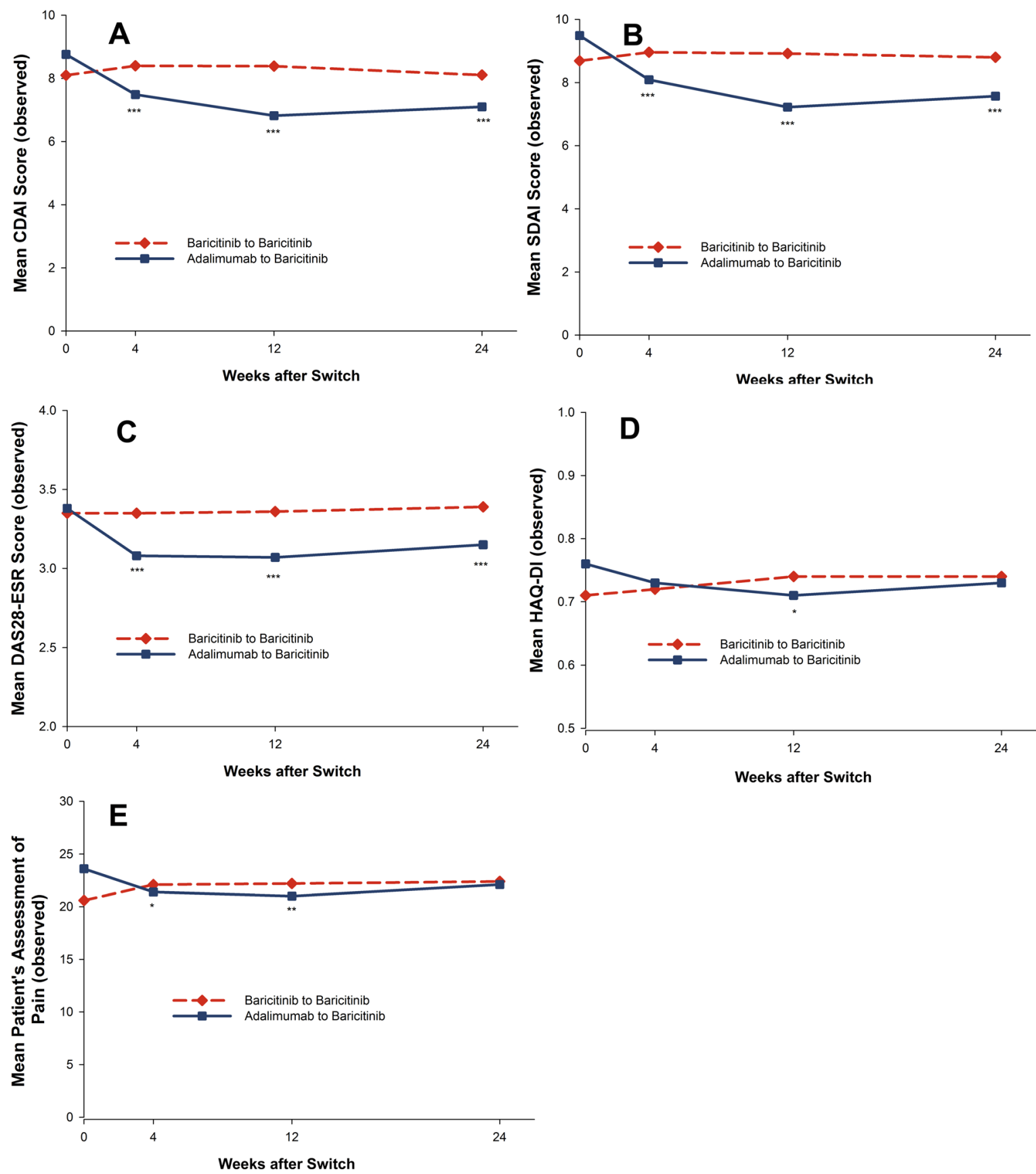

Figure 2 Mean observed values for CDAI (A), SDAI (B), DAS28-ESR (C), physical function (HAQ-DI) (D) and patient's assessment of pain (E) through 24 weeks in the switched patients from RA-BEYOND. ${ }^{*} p \leq 0.05 ;{ }^{* *} p \leq 0.01 ;{ }^{* * *} p \leq 0.001$ for within-group change based on mixed model for repeated measures. Week $0=$ time of switch (or entry to RA-BEYOND). CDAl, clinical disease activity index; DAS28-ESR, disease activity score using 28-joint count with erythrocyte sedimentation rate; HAQ-DI, Health Assessment Questionnaire Disability index; SDAI, simplified disease activity index.

present dataset is of relevance as it may better approximate real-world practice in that patients with inadequate response to adalimumab in RA-BEAM who were rescued to baricitinib at a scheduled visit did so only 2 weeks following the last dose of adalimumab. Unsurprisingly, given the prior findings of RA-BEACON, these patients showed clinical improvement with baricitinib (although as noted below, present observations are limited by the open-label nature of rescue). Perhaps the more practically important novel finding from the present study is that adverse event data from the period immediately following the treatment change suggest that a prompt transition from adalimumab to baricitinib can be executed with acceptable safety and tolerability.
Switching between therapeutics may occur even in patients who exhibit clinical responses and tolerate a given initial treatment. Patients randomised to adalimumab who completed the 52 week RA-BEAM without requiring rescue were switched to baricitinib in RA-BEYOND, with maintained efficacy and acceptable safety. These findings are of practical clinical relevance where patients receiving a biologic TNF inhibitor who have not necessarily exhibited intolerance or poor response to that agent are contemplating transition to baricitinib. This scenario could arise, for instance, due to patient preference for oral over injectable treatment options. ${ }^{6}$ As patients communicate with their healthcare providers regarding preferred treatments, they can be assured that important quality-of-life outcomes will 


\section{Baricitinib to Baricitinib}
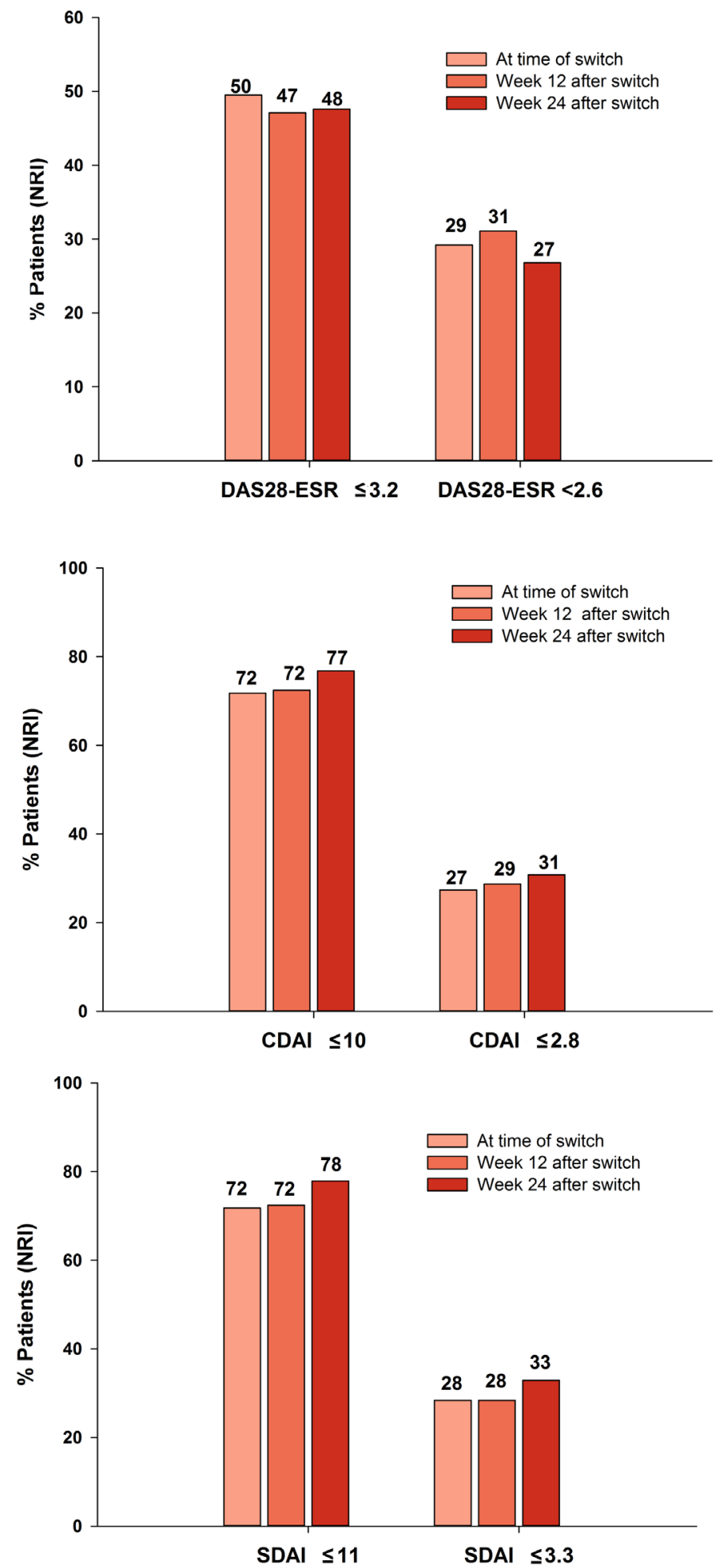

Adalimumab to Baricitinib
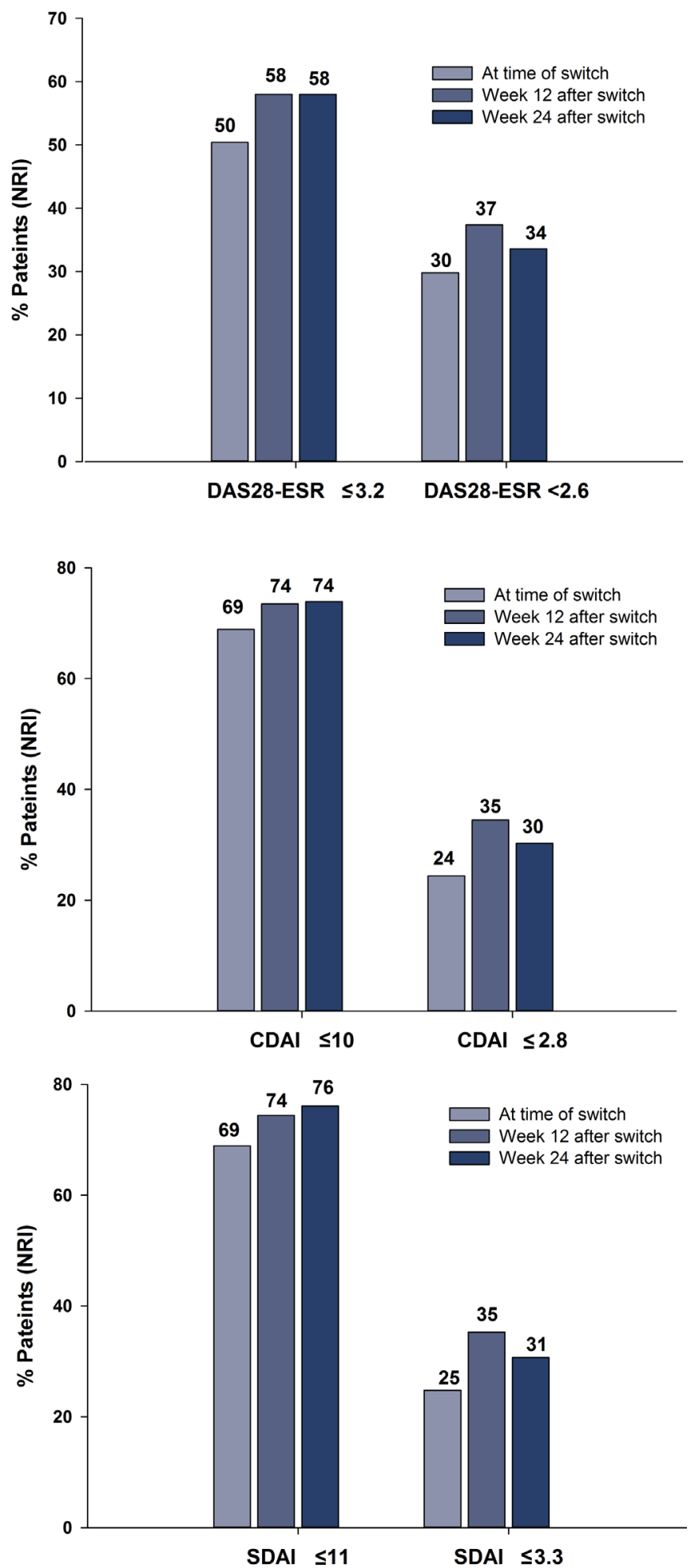

Figure 3 Low disease activity and remission at weeks 0,12 and 24 after switch in switched patients from RA-BEYOND. Week $0=$ time of switch (or entry to RA-BEYOND). NRI was used for missing data imputation. CDAl, clinical disease activity index; DAS28-ESR, disease activity score using 28-joint count with erythrocyte sedimentation rate; NRI, non-responder imputation; SDAl, simplified disease activity index.

be maintained after switching to baricitinib as physical function and pain improvements that occurred in RA-BEAM with adalimumab were maintained into the LTE after switching to baricitinib.
The switch from adalimumab to baricitinib occurred without a washout period, and adalimumab has a mean circulating halflife of approximately 14 days. ${ }^{9}$ Patients would therefore have received several weeks of dual TNF and JAK1/JAK2 inhibition 
Table 3 Safety in rescued patients from RA-BEAM and patients switched in RA-BEYOND

\begin{tabular}{|c|c|c|c|c|}
\hline & \multicolumn{2}{|c|}{$\begin{array}{l}\text { Rescued patients in RA-BEAM } \\
\text { ( } 24 \text { weeks after rescue) }\end{array}$} & \multicolumn{2}{|c|}{$\begin{array}{l}\text { Switched patients in RA-BEYOND } \\
\text { (weeks 0-24 after switch) }\end{array}$} \\
\hline & $\begin{array}{l}\text { Baricitinib to baricitinib } \\
(\mathrm{n}=35, \mathrm{PYE}=15.8)\end{array}$ & $\begin{array}{l}\text { Adalimumab to baricitinib } \\
(\mathrm{n}=40, \mathrm{PYE}=19.6)\end{array}$ & $\begin{array}{l}\text { Baricitinib to baricitinib } \\
(\mathrm{n}=381, \mathrm{PYE}=189.4)\end{array}$ & $\begin{array}{l}\text { Adalimumab to baricitinib } \\
(\mathrm{n}=238, \mathrm{PYE}=118.2)\end{array}$ \\
\hline Infections & $11(31.4)(69.6)$ & $7(17.5)(35.6)$ & $87(22.8)(45.9)$ & $49(20.6)(41.5)$ \\
\hline Herpes zoster & $1(2.9)(6.3)$ & $1(2.5)(5.1)$ & $5(1.3)(2.6)$ & $5(2.1)(4.2)$ \\
\hline Patients with $\geq 1 \mathrm{SAE}$ & $6(17.1)(37.9)$ & $2(5.0)(10.2)$ & $26(6.8)(13.7)$ & $11(4.6)(9.3)$ \\
\hline Serious infections & 0 & 0 & $8(2.1)(4.2)$ & 4 (1.7) (3.4) \\
\hline
\end{tabular}

Data are $\mathrm{n}(\%)$ (EAIR).

*Gastrointestinal disorders included nausea, dental caries, constipation, gastritis, vomiting, diarrhoea, inguinal hernia, mouth ulceration and upper gastrointestinal haemorrhage. †Gastrointestinal disorders included dyspepsia, abdominal pain, constipation, stomatitis, upper abdominal pain, diarrhoea, enterocolitis and hyperchlorhydria.

$A E$, adverse event; EAIR, exposure-adjusted incidence rate; PYE, patient-years of exposure; SAE, serious adverse event; TEAE, treatment-emergent adverse event.

after treatment change. Hence, although the observed improvements in CDAI and SDAI were sustained over 24 weeks (figure 2) and may simply reflect the improved efficacy seen with baricitinib over adalimumab in the randomised period of RA-BEAM, some of the initial benefit evident after switch might in fact be accounted for by the combination. From a biological perspective, there are reasons to consider that a combination of TNF and JAK inhibition could have merit. TNF inhibition using bDMARDs provides near-complete, sustained inhibition of the TNF pathway and its downstream effects. Baricitinib, on the other hand, provides partial and transient inhibition of the broader variety of JAK1/JAK2-dependent pathways. Accordingly, whole blood microarray evaluation in RA-BEAM has shown that baricitinib and adalimumab exert pharmacodynamic effects that impact substantially non-overlapping sets of mediators. ${ }^{10}$ The observation of added efficacy, without apparent acute safety signal during the weeks when patients were exposed to both adalimumab and baricitinib, is of interest given the findings from earlier, dedicated combination targeted DMARD studies, which showed that compared with single targeted therapy, selected combinations (TNF inhibition with either interleukin-1 or T-cell costimulation blockade) increased safety risks without adding benefit. $^{1112}$

The current analysis has a number of relevant limitations. First, although initial randomised treatment assignment remained blinded at all times, baricitinib was administered in an open-label manner after rescue or switch. This likely influenced subjective responses thereafter, in particular for RA-BEAM rescue, where patients knew they were moving to baricitinib from a treatment that was ineffective for them, and which could have been placebo. Given these dynamics, the psychological effects of 'rescue' may have masked detectable differences between groups based on prior treatment. This could account for the counterintuitive finding that, in contrast to acute phase markers, which significantly improved only in those patients rescued from adalimumab to baricitinib, improvements in composite disease activity scores (which include subjective components) after rescue to openlabel baricitinib did not appear different for patients rescued from blinded baricitinib (ie, no treatment change, apart from background therapy) compared with those rescued from blinded adalimumab (ie, a change of treatment and a setting (TNF-IR) where baricitinib has been proven to be efficacious). ${ }^{8}$ This bias was likely less of a factor for the switch to baricitinib on entering the LTE, as at the time of switch, all patients were knowingly receiving active treatment to which they had exhibited a reasonable response; the psychological placebo effect of rescue from an ineffective treatment was not in play. In addition, rescue criteria in RA-BEAM differed during the period of interest; at week 16, patients were automatically rescued without at least a minimal improvement in swollen/tender joint counts, whereas thereafter, investigators decided whether to rescue. Finally, the transitions to baricitinib (rescue and switch) were not randomised.

In conclusion, these data support and extend the findings of prior baricitinib studies, ${ }^{68}$ that in patients with RA, transition from adalimumab to baricitinib does not require lengthy washout from the prior treatment and is associated with maintained clinical disease control, with acceptable safety.

\section{Author affiliations \\ ${ }^{1}$ The First Department of Internal Medicine, School of Medicine, University of Occupational and Environmental Health, Japan, Kitakyushu, Japan \\ ${ }^{2}$ Dept of Rheumatology, Sorbonne Universite, Pierre Louis Institute of Epidemiology and Public Health, Pépites team; APHP, GH Pitie Salpetriere, Paris, France \\ ${ }^{3}$ Rebecca MacDonald Centre for Arthritis and Autoimmune Diseases, Mount Sinai Hospital, Toronto, Ontario, Canada \\ ${ }^{4}$ University of Toronto, Toronto, Ontario, Canada \\ ${ }^{5}$ Eli Lilly and Company, Indianapolis, Indiana, USA \\ ${ }^{6}$ Botnar Research Centre, Nuffield Department of Orthopaedics, Rheumatology and Musculoskeletal Sciences, University of Oxford, Oxford, United Kingdom}

Acknowledgements The authors thank Kathy Oneacre, MA, of Syneos Health for medical writing support and assistance with preparation and submission of this article. PCT would like to acknowledge support by the National Institute for Health Research Oxford Biomedical Research Centre and by AR UK. This article is based on work presented at the 2016 ACR/ARHP Annual Meeting: Taylor PC, Keystone E, Ortmann R, Issa M, Xie L, Muram D, Bradley JD, de Bono S, Rooney T, Tanaka Y. Efficacy and Safety of Switching from Adalimumab to Baricitinib: Phase 3 Data in Patients with Rheumatoid Arthritis (abstract). Arthritis Rheumatol. 2016; 68 (suppl 10) and at the 2018 ACR/ARHP Annual Meeting: Weinblatt ME, Taylor PC, Keystone EC, Ortmann RA, Issa M, Xie L, de Bono S, Tanaka Y. Efficacy and Safety of Switching from Adalimumab to Baricitinib: Long-Term Data from Phase 3 Extension Study in Patients with Rheumatoid Arthritis (abstract). ${ }^{10}$ Arthritis Rheumatol. 2018; 70 (suppl 10).

Contributors YT participated in the conception of the work, the interpretation of data for the work and the critical revision of the manuscript for important intellectual content; BF participated in the interpretation of data for the work and the critical revision of the manuscript for important intellectual content; EK, RO, LX and BZ participated in in analysis and interpretation of data for the work and critical revision of the manuscript for important intellectual content; MI participated in the conception and design of the work, acquisition, analysis and interpretation of data for the work, drafting of the manuscript, and critical revision of the manuscript for important intellectual content. HP participated in analysis and interpretation of data for the work and drafting of the manuscript; CG participated in the acquisition and interpretation of data for the work and in the drafting of the manuscript 
for important intellectual content; SDB participated in the design, analysis and interpretation of data for the work and in the critical revision of the manuscript for important intellectual content; TR participated in the acquisition, analysis and interpretation of data for the work and in the critical revision of the manuscript for important intellectual content; PCT participated in the conception and design of the work, interpretation of data for the work, and drafting and critical revision of the manuscript for important intellectual content.

Funding The studies were sponsored by Eli Lilly and Company and Incyte Corporation.

Competing interests $\mathrm{YT}$ has received speaking fees and/or honoraria from Daiichi-Sankyo, Astellas, Eli Lilly, Chugai, Sanofi, Abbvie, Pfizer, YL Biologics, BristolMyers, Glaxo-Smithkline, UCB, Mitsubishi-Tanabe, Novartis, Eisai, Takeda, Janssen and Asahi-kasei, and has received research grants from Mitsubishi-Tanabe, BristolMyers, Eisai, Chugai, Takeda, Abbvie, Astellas, Daiichi-Sankyo, Ono, MSD and TaishoToyama; BF reports grants and personal fees from AbbVie, Eli Lilly, Pfizer and MSD, and personal fees from Biogen, BMS, Celgene, Janssen, Medac, Nordic, Novartis, Roche, SOBI, Sanofi-Genzyme and UCB outside the submitted work; EK reports funding for research from AbbVie, Amgen, Bristol-Myers Squibb, F. Hoffmann-La Roche Inc, Gilead, Janssen, Lilly Pharmaceuticals, Pfizer Pharmaceuticals, SanofiAventis, consulting agreements with AbbVie, Amgen, AstraZeneca Pharma, Biotest, Bristol-Myers Squibb Company, Celltrion, Crescendo Bioscience, F. Hoffmann-La Roche Inc, Genentech Inc, Gilead, Janssen Inc, Lilly Pharmaceuticals, Merck, Pfizer Pharmaceuticals, Sandoz and UCB, and has received speaker honoraria from Amgen, AbbVie, Bristol-Myers Squibb Canada, F. Hoffmann-La Roche Inc., Janssen, Merck, Pfizer Pharmaceuticals, Sanofi Genzyme and UCB; RO, LX, BZ, MI, HP, CG, SDB and TR are employees and stockholders of Eli Lilly and Company; PCT reports grants from Eli Lilly and Company, Celgene, UCB and Galapagos, and personal fees from Eli Lilly and Company, AbbVie, Gilead, and Pfizer during the conduct of the study.

Patient consent for publication Not required.

Provenance and peer review Not commissioned; externally peer reviewed.

Open access This is an open access article distributed in accordance with the Creative Commons Attribution Non Commercial (CC BY-NC 4.0) license, which permits others to distribute, remix, adapt, build upon this work non-commercially, and license their derivative works on different terms, provided the original work is properly cited, appropriate credit is given, any changes made indicated, and the use is non-commercial. See: http://creativecommons.org/licenses/by-nc/4.0/.

\section{REFERENCES}

1 Smolen JS, Aletaha D, McInnes IB. Rheumatoid arthritis. The Lancet 2016;388:2023-38

2 Smolen JS, Breedveld FC, Burmester GR, et al. Treating rheumatoid arthritis to target: 2014 update of the recommendations of an international Task Force. Ann Rheum Dis 2016;75:3-15.

3 Smolen JS, Landewé R, Bijlsma J, et al. EULAR recommendations for the management of rheumatoid arthritis with synthetic and biological disease-modifying antirheumatic drugs: 2016 update. Ann Rheum Dis 2017;76:960-77.

4. Fridman JS, Scherle PA, Collins R, et al. Selective inhibition of JAK1 and Jak2 is efficacious in rodent models of arthritis: preclinical characterization of INCB028050 J Immunol 2010;184:5298-307.

5 O'Shea JJ, Holland SM, Staudt LM. JAKs and STATs in immunity, immunodeficiency, and cancer. N Engl J Med 2013;368:161-70.

6 Taylor PC, Keystone EC, van der Heijde D, et al. Baricitinib versus placebo or adalimumab in rheumatoid arthritis. N Engl J Med Overseas Ed 2017;376:652-62.

7 Emery P. Optimizing outcomes in patients with rheumatoid arthritis and an inadequate response to anti-TNF treatment. Rheumatology 2012;51(suppl 5):v22-30

8. Genovese $\mathrm{MC}$, Kremer J, Zamani O, et al. Baricitinib in patients with refractory rheumatoid arthritis. N Engl J Med 2016;374:1243-52.

9 Anon. Humira (Adalimumab) lpackage insert. North Chicago, IL: AbbVie Inc, 2017.

10 Emery P, Taylor PC, Weinblatt M, et al. Microarray Pathway Analysis Comparing Baricitinib and Adalimumab in Moderate to Severe Rheumatoid Arthritis Patients, from a Phase 3 Study [abstract]. Arthritis Rheumatol 2017;69(suppl 10).

11 Genovese MC, Cohen S, Moreland L, et al. Combination therapy with etanercept and anakinra in the treatment of patients with rheumatoid arthritis who have been treated unsuccessfully with methotrexate. Arthritis Rheum 2004:50:1412-9.

12 Weinblatt M, Schiff M, Goldman A, et al. Selective costimulation modulation using abatacept in patients with active rheumatoid arthritis while receiving etanercept: a randomised clinical trial. Ann Rheum Dis 2007;66:228-34. 